\title{
CURVATURE DEPENDENT ENERGY OF SURFACE CURVES IN MINKOWSKI SPACE
}

\author{
TALAT KORPINAR ${ }^{1}$, RIDVAN CEM DEMIRKOL ${ }^{1, *}$ AND VEDAT ASIL $^{2}$ \\ ${ }^{1}$ Mus Alparslan University, Department of Mathematics, 49250, Mus, Turkey \\ ${ }^{2}$ Firat University, Department of Mathematics, 23100, Elazig, Turkey \\ *Corresponding author: rcdemirkol@gmail.com
}

\begin{abstract}
In this paper, we firstly introduce kinematics properties of the moving particle lying on a surface $\mathbf{S}$. We assume that the particle corresponds to a different type of surface curves such that they are characterized by using the Darboux vector field $\mathbf{W}$ in Minkowski spacetime. Based on this result, we present geometrical understanding of the energy of the particle in each Darboux vector fields whether they lie on a spacelike surface or a timelike surface. Then, we also determine the bending elastic energy functional for the same particle on a surface $\mathbf{S}$ by assuming the particle has a bending feature of elastica. Finally, we prove that bending energy formula can be represented by the energy of the particle in each Darboux vector field
\end{abstract} W.

\section{INTRODUCTION}

The study of computing an energy for a given vector field depending on the structure of the geometrical spaces has earned such attention in the last couple years. It has been shown that tis type of computations has numerous applications in various fields. Thus, multidisciplinary subjects have been evolved. For instance, Wood [1] studied energy on the unit vector field firstly. Gil-Medrano [2] worked on a relation between energy and volume of vector fields. Chacon et al. in [3], [4] investigated the energy distribution and corrected energy

Received 2017-10-17; accepted 2017-12-16; published 2018-03-07.

2010 Mathematics Subject Classification. 53C41, 53A10.

Key words and phrases. energy; Minkowski Space; Darboux vector field; surface curve.

(C)2018 Authors retain the copyrights of their papers, and all open access articles are distributed under the terms of the Creative Commons Attribution License. 
of distributions on Riemannian manifolds. Altin [5] computed the energy of Frenet vector fields for given non-lightlike curves.

The corresponding theory for the functional of curvature-based energy is considered to be at its early stages of evolution. Some prolific fields and pioneering studies for this theory can be found in mathematical physics, membrane chemistry, computer-aided geometric design and geometric modelling, shell engineering, biology and thin plate ( $[6],[7],[8],[9],[10])$. Darboux frame is considered as a natural moving frame. It is implemented in surface geometry to characterize features of the curve lying on the surface. It is also used to understand characterization of functionals, which play a significant role to construct surface curves. Some of the well-known functionals and related works can be given as follows. Bending energy functional has appeared firstly Bernoulli-Euler elastica formulation for energy [11]. MVS energy functional is used for aesthetic surface design [12]. MVS cross energy functional computes the deviation of the surface from a cylinder or a perfect sphere [13]. The energy of infinitesimally small surface area can be defined by average energy of surface curves which pass through that surface. Thus, we work on curves lying on appropriate surfaces to compute these functionals. Moreover, total surface energy can be obtained by integrating the energy of the small area element over the entire surface [14].

In this study, we compute energy on the moving particle lying on the surface which is defined in Minkowski spacetime. Minkowski spacetime has a close connection between mass-energy and motion-energy concept, which are topics of special relativity ( [15], [16], [17], [18]). Furthermore, we aim to present usefulness of geometrical perspective on the computation of the energy by calculating curvature-based energy for surface curves. Moreover, we introduce the relation between energy on surface curves in each Darboux vector field W and curvature-based bending energy functional. The method we use for computing the energy of Darboux vector fields is that we consider a vector field as a map from manifold $M$ to the Riemannian manifold $\left(T M, \rho_{s}\right)$, where $T M$ is tangent bundle of a Riemannian manifold and $\rho_{s}$ is a Sasaki metric induced from $T M$ naturally.

The designation of the paper is as follows. We firstly present fundamental definitions of Darboux frame equations for different types of surface curves in Minkowski space. Then we give a geometrical interpretation of the energy for unit vector fields. Guided by this fundamental information, we compute energy of the moving particle corresponding to a surface curve in Minkowski space.

\section{Kinematics of the Particle on a Surface}

Let $\Gamma$ be a particle moving on a surface $\mathcal{S}$ such that the precise location of the particle is specified by $\Gamma=\Gamma(t)$, where $t$ is a time parameter. Changing the time parameter describes the motion and trajectory. Thus, the trajectory corresponds to a curve $\zeta$ in the surface for a moving particle. It is convenient to remind the arc-length parameter $s$, which is used to compute the distance traveled by a particle along its trajectory. 
It is defined by

$$
\frac{d s}{d t}=\|\mathbf{v}\|
$$

where $\mathbf{v}=\mathbf{v}(t)=\frac{d \zeta}{d t}$ is the velocity vector and $\frac{d \zeta}{d t} \neq 0$. In particle dynamics, the arc-length parameter $s$ is considered as a function of $t$. Thanks to the arc-length, it is also determined Serret-Frenet frame, which allows us determining the characterization of the intrinsic geometrical features of the regular curve. This coordinate system is constructed by three orthonormal vectors $\mathbf{e}_{(\alpha)}$ and the curve $\zeta$ itself, assuming the curve is sufficiently smooth at each point. The index within the parenthesis is the tetrad index that describes a particular member of the tetrad. In particular, $\mathbf{e}_{(0)}$ is the unit tangent vector, $\mathbf{e}_{(1)}, \mathbf{e}_{(2)}$ is the unit normal and binormal vector of the curve $\zeta$, respectively. Orthonormality conditions are summarized by $\mathbf{e}_{(\alpha)} \mathbf{e}_{(\beta)}=\eta_{\alpha \beta}$, where $\eta_{\alpha \beta}$ is a Euclidean metric such that: $\operatorname{diag}(1,1,1)$. Thus, we have the following formulas for the Frenet frame equations.

$$
\begin{aligned}
\frac{D \mathbf{e}_{(0)}}{d s} & =\kappa \mathbf{e}_{(1)}, \\
\frac{D \mathbf{e}_{(1)}}{d s} & =-\kappa \mathbf{e}_{(0)}+\tau \mathbf{e}_{(2)}, \\
\frac{D \mathbf{e}_{(2)}}{d s} & =-\tau \mathbf{e}_{(1)},
\end{aligned}
$$

where $\kappa$ and $\tau$ are curvature and torsion of the curve, respectively.

In addition to Frenet frame, it can be defined a new frame called as Darboux frame on the oriented surface S. For the trajectory of the moving particle, which corresponds to a curve $\zeta$ on the surface, the Darboux vectors $\mathbf{e}_{(0)}, \mathbf{n}, \mathbf{P}=\mathbf{e}_{(\mathbf{0})} \times \mathbf{n}$, are defined. They are the unit tangent of the curve, unit normal of the surface, and normal of the tangent, respectively. They satisfy following equations and properties.

Case 1. Let $\mathbf{S}$ be an oriented spacelike surface and moving particle $\Gamma$ lying on $\mathbf{S}$ has a unit spacelike tangent vector $\mathbf{e}_{(0)}$, then we have

$$
\begin{aligned}
\frac{D \mathbf{e}_{(0)}}{d s} & =\kappa_{g} \mathbf{P}+\kappa_{n} \mathbf{n}, \\
\frac{D \mathbf{P}}{d s} & =-\kappa_{g} \mathbf{e}_{(0)}+\tau_{g} \mathbf{n}, \\
\frac{D \mathbf{n}}{d s} & =\kappa_{n} \mathbf{e}_{(0)}+\tau_{g} \mathbf{P},
\end{aligned}
$$

where $\kappa_{g}, \kappa_{n}, \tau_{g}$ are geodesic curvature, normal curvature and geodesic torsion of the curve, [19].

Case 2. Let $\mathbf{S}$ be an oriented timelike surface and moving particle lying on $\mathbf{S}$ has a unit spacelike tangent vector $\mathbf{e}_{(0)}$, then we have 


$$
\begin{aligned}
\frac{D \mathbf{e}_{(0)}}{d s} & =\kappa_{g} \mathbf{P}-\kappa_{n} \mathbf{n}, \\
\frac{D \mathbf{P}}{d s} & =\kappa_{g} \mathbf{e}_{(0)}+\tau_{g} \mathbf{n}, \\
\frac{D \mathbf{n}}{d s} & =\kappa_{n} \mathbf{e}_{(0)}+\tau_{g} \mathbf{P},
\end{aligned}
$$

where $\kappa_{g}, \kappa_{n}, \tau_{g}$ are geodesic curvature, normal curvature and geodesic torsion of the curve, [19].

Case 3. Let $\mathbf{S}$ be an oriented timelike surface and moving particle lying on $\mathbf{S}$ has a unit timelike tangent vector $\mathbf{e}_{(0)}$, then we have

$$
\begin{aligned}
\frac{D \mathbf{e}_{(0)}}{d s} & =\kappa_{g} \mathbf{P}+\kappa_{n} \mathbf{n}, \\
\frac{D \mathbf{P}}{d s} & =\kappa_{g} \mathbf{e}_{(0)}-\tau_{g} \mathbf{n}, \\
\frac{D \mathbf{n}}{d s} & =\kappa_{n} \mathbf{e}_{(0)}+\tau_{g} \mathbf{P},
\end{aligned}
$$

where $\kappa_{g}, \kappa_{n}, \tau_{g}$ are geodesic curvature, normal curvature and geodesic torsion of the curve, [19].

Since we identify $\mathbf{e}_{(0)}$ as a unit vector as a tangent to the curve at each point on the curve, we have $\mathbf{e}_{(0)}=d \Gamma^{u} / d s$, where $\Gamma^{u}$ is the point on the trajectory of curve $\zeta$. Thus $\mathbf{e}_{(0)}, \mathbf{P}$ and $\mathbf{n}$ generate the Darboux frame $\mathbf{W}$ and Equation 2.1, 2.2, and 2.3 are known as Darboux equations for each case.

\section{Energy on the Unit Vector Fields in Space}

We first give the fundamental definitions and propositions which are used to compute the energy of the unit vector field.

Definition 3.1. For two Riemannian manifolds $(M, \rho)$ and $(N, h)$ the energy of a differentiable map $f$ : $(M, \rho) \rightarrow(N, h)$ can be defined as

$$
\operatorname{Energy}(f)=\frac{1}{2} \int_{M} \sum_{a=1}^{n} h\left(d f\left(e_{a}\right), d f\left(e_{a}\right)\right) v,
$$

where $\left\{e_{a}\right\}$ is a local basis of the tangent space and $v$ is the canonical volume form in $M$ [1].

Proposition 3.1. Let $Q: T\left(T^{1} M\right) \rightarrow T^{1} M$ be the connection map. The following two conditions hold:

i) $\omega \circ Q=\omega \circ d \omega$ and $\omega \circ Q=\omega \circ \tilde{\omega}$, where $\tilde{\omega}: T\left(T^{1} M\right) \rightarrow T^{1} M$ is the tangent bundle projection;

ii) for $\varrho \in T_{x} M$ and a section $\xi: M \rightarrow T^{1} M$; we have

$$
Q(d \xi(\varrho))=D_{\varrho} \xi
$$

where $D$ is the Levi-Civita covariant derivative [1]. 
Definition 3.2. For $\varsigma_{1}, \varsigma_{2} \in T_{\xi}\left(T^{1} M\right)$, we define

$$
\rho_{S}\left(\varsigma_{1}, \varsigma_{2}\right)=\rho\left(d \omega\left(\varsigma_{1}\right), d \omega\left(\varsigma_{2}\right)\right)+\rho\left(Q\left(\varsigma_{1}\right), Q\left(\varsigma_{2}\right)\right) .
$$

This yields a Riemannian metric on TM. As known $\rho_{S}$ is called the Sasaki metric that also makes the projection $\omega: T^{1} M \rightarrow M$ a Riemannian submersion.

\section{Bending Energy Functional by Darboux Vector Fields}

In the theory of relativity, all the energy moving through an object contributes to the total mass of the body that measures how much it can resist to acceleration. Each kinetic and potential energy makes a highly proportional contribution to the mass [20], [21], [22], [23] . In this study not only we compute the energy on surface curves but we also investigate its close correlation with bending energy of elastica which is a variational problem proposed firstly by Daniel Bernoulli to Leonard Euler in 1744. Euler bending elastic energy formula for a space curve in the 3-dimensional Frenet curvature along the curve is known as

$$
H_{B}=\frac{1}{2} \int\left\|D_{\mathbf{e}_{(0)}} \mathbf{e}_{(0)}\right\|^{2} d s
$$

where $s$ is an arclength, [10]. Furthermore, we know that geodesic curvature and normal curvature are not independent and sum of their squares on a minimal surface gives

$$
\mathcal{K}_{G}=-\left(\kappa_{n}^{2}+\tau_{g}^{2}\right)
$$

where $\mathcal{K}_{G}$ is Gaussian curvature [10].

Case 1. Let $\mathbf{S}$ be an oriented spacelike surface and moving particle lying on $\mathbf{S}$ has a unit spacelike tangent vector $\mathbf{e}_{(0)}$

Theorem 4.1. Let $\Gamma$ be a moving particle on surface $S$ such that it corresponds to a curve $\zeta$. Then, energy on the particle in tangent vector field by using Sasaki metric is stated by

$$
\text { Energye }_{(0)}=\frac{1}{2}\left(s+\int_{0}^{s}\left(\kappa_{g}^{2}-\kappa_{n}^{2}\right) d s\right) .
$$

Proof. From Equation 3.1 and 3.2 we know

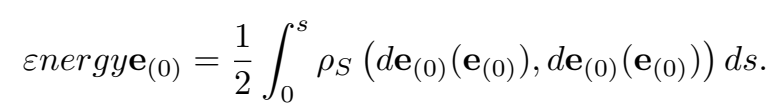

Using also Equation 3.3 we have

$$
\begin{gathered}
\rho_{S}\left(d \mathbf{e}_{(0)}\left(\mathbf{e}_{(0)}\right), d \mathbf{e}_{(0)}\left(\mathbf{e}_{(0)}\right)\right)=\rho\left(d \omega\left(\mathbf{e}_{(0)}\left(\mathbf{e}_{(0)}\right)\right), d \omega\left(\mathbf{e}_{(0)}\left(\mathbf{e}_{(0)}\right)\right)\right) \\
+\rho\left(Q\left(\mathbf{e}_{(0)}\left(\mathbf{e}_{(0)}\right)\right), Q\left(\mathbf{e}_{(0)}\left(\mathbf{e}_{(0)}\right)\right)\right) .
\end{gathered}
$$


Since $\mathbf{e}_{(0)}$ is a section, we get

$$
d(\omega) \circ d\left(\mathbf{e}_{(0)}\right)=d\left(\omega \circ \mathbf{e}_{(0)}\right)=d\left(i d_{C}\right)=i d_{T C} .
$$

We also know

$$
Q\left(\mathbf{e}_{(0)}\left(\mathbf{e}_{(0)}\right)\right)=D_{\mathbf{e}_{(0)}} \mathbf{e}_{(0)}=\kappa_{g} \mathbf{P}+\kappa_{n} \mathbf{n}
$$

Thus, we find from the Equation 2.1

$$
\begin{aligned}
\rho_{S}\left(d \mathbf{e}_{(0)}\left(\mathbf{e}_{(0)}\right), d \mathbf{e}_{(0)}\left(\mathbf{e}_{(0)}\right)\right) & =\rho\left(\mathbf{e}_{(0)}, \mathbf{e}_{(0)}\right)+\rho\left(D_{\mathbf{e}_{(0)}} \mathbf{e}_{(0)}, D_{\mathbf{e}_{(0)}} \mathbf{e}_{(0)}\right) \\
& =1+\kappa_{g}^{2}-\kappa_{n}^{2}
\end{aligned}
$$

So we can easily obtain

$$
\operatorname{\varepsilon nergye}_{(0)}=\frac{1}{2}\left(s+\int_{0}^{s}\left(\kappa_{g}^{2}-\kappa_{n}^{2}\right) d s\right) .
$$

This completes the proof.

Conclusion 4.1. Let $\zeta$ be a spacelike curve lying on spacelike surface $\mathbf{S}$. Then we have $\left\|D_{\mathbf{e}_{(0)}} \mathbf{e}_{(0)}\right\|^{2}=\kappa_{g}^{2}-\kappa_{n}^{2}$. Thus, we obtain following relation for the bending energy of elastica:

$$
H_{B}=\text { energy } \mathbf{e}_{(0)}-\frac{1}{2} s .
$$

Proof. It is obvious from the Equation 4.1 and Theorem 4.1.

Theorem 4.2. Let $\Gamma$ be a moving particle on surface $S$ such that it corresponds to a curve $\zeta$. Then, energy on the particle in vector field of the normal of the surface by using Sasaki metric is stated by

$$
\operatorname{Energy}(\mathbf{n})=\frac{1}{2}\left(s+\int_{0}^{s}\left(\kappa_{n}^{2}+\tau_{g}^{2}\right) d s\right) .
$$

Proof. If we follow the similar steps as in the Theorem 4.1, the proof is obvious.

Conclusion 4.2. Let $\zeta$ be a spacelike curve lying on spacelike surface S. Then we have for a Gaussian curvature $K_{G}$

$$
\operatorname{Energy}(\mathbf{n})=\frac{1}{2}\left(s-\int_{0}^{s} \mathcal{K}_{G} d s\right) .
$$

Proof. It is obvious from Equation 4.1 and Theorem 4.2. 
Theorem 4.3. Let $\Gamma$ be a moving particle on surface $S$ such that it corresponds to a curve $\zeta$. Then, energy on the particle in tangent's normal vector field by using Sasaki metric is stated by

$$
\operatorname{\varepsilon nergy}(\mathbf{P})=\frac{1}{2}\left(s+\int_{0}^{s}\left(\kappa_{g}^{2}-\tau_{g}^{2}\right) d s\right) .
$$

Proof. If we follow the similar steps as in the Theorem 4.1, the proof is obvious.

Case 2. Let $\mathbf{S}$ be an oriented timelike surface and moving particle lying on $\mathbf{S}$ has a unit spacelike tangent vector $\mathbf{e}_{(0)}$.

Theorem 4.4. Let $\Gamma$ be a moving particle on surface $S$ such that it corresponds to a curve $\zeta$. Then, energy on the particle in tangent vector field by using Sasaki metric is stated by

$$
\text { Energye }_{(0)}=\frac{1}{2}\left(s+\int_{0}^{s}\left(-\kappa_{g}^{2}+\kappa_{n}^{2}\right) d s\right) .
$$

Proof. From Equation 3.1 and 3.2 we know

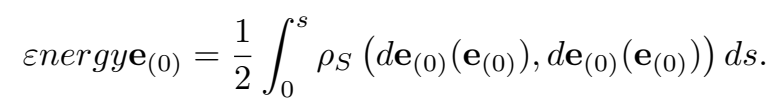

Using the Equation 3.3 and knowing $\mathbf{e}_{(0)}$ is a section we obtain that

$$
\begin{gathered}
\rho_{S}\left(d \mathbf{e}_{(0)}\left(\mathbf{e}_{(0)}\right), d \mathbf{e}_{(0)}\left(\mathbf{e}_{(0)}\right)\right)=\rho\left(d \omega\left(\mathbf{e}_{(0)}\left(\mathbf{e}_{(0)}\right)\right), d \omega\left(\mathbf{e}_{(0)}\left(\mathbf{e}_{(0)}\right)\right)\right) \\
+\rho\left(Q\left(\mathbf{e}_{(0)}\left(\mathbf{e}_{(0)}\right)\right), Q\left(\mathbf{e}_{(0)}\left(\mathbf{e}_{(0)}\right)\right)\right)
\end{gathered}
$$

and

$$
d(\omega) \circ d\left(\mathbf{e}_{(0)}\right)=d\left(\omega \circ \mathbf{e}_{(0)}\right)=d\left(i d_{C}\right)=i d_{T C} .
$$

It is also true that

$$
Q\left(\mathbf{e}_{(0)}\left(\mathbf{e}_{(0)}\right)\right)=D_{\mathbf{e}_{(0)}} \mathbf{e}_{(0)}=\kappa_{g} \mathbf{P}-\kappa_{n} \mathbf{n}
$$

Moreover we find from the Equation 2.2

$$
\begin{aligned}
\rho_{S}\left(d \mathbf{e}_{(0)}\left(\mathbf{e}_{(0)}\right), d \mathbf{e}_{(0)}\left(\mathbf{e}_{(0)}\right)\right) & =\rho\left(\mathbf{e}_{(0)}, \mathbf{e}_{(0)}\right)+\rho\left(D_{\mathbf{e}_{(0)}} \mathbf{e}_{(0)}, D_{\mathbf{e}_{(0)}} \mathbf{e}_{(0)}\right) \\
& =1-\kappa_{g}^{2}+\kappa_{n}^{2} .
\end{aligned}
$$

Thus we can easily obtain

$$
\text { Energye }_{(0)}=\frac{1}{2}\left(s+\int_{0}^{s}\left(-\kappa_{g}^{2}+\kappa_{n}^{2}\right) d s\right) .
$$

Conclusion 4.3. Let $\zeta$ be a spacelike curve lying on timelike surface $\mathbf{S}$. Then we have $\left\|D_{\mathbf{e}_{(0)}} \mathbf{e}_{(0)}\right\|^{2}=$ $-\kappa_{g}^{2}+\kappa_{n}^{2}$. Thus, we obtain following relation for the bending energy of elastica:

$$
H_{B}=\text { energy }_{(0)}-\frac{1}{2} s .
$$


Proof. It is obvious from the Equation 4.1 and Theorem 4.4.

Theorem 4.5. Energy on the moving particle in surface's normal and tangent's normal vector field by using Sasaki metric is stated by

$$
\begin{aligned}
& \operatorname{\varepsilon nergy}(\mathbf{n})=\frac{1}{2}\left(s+\int_{0}^{s}\left(\kappa_{n}^{2}-\tau_{g}^{2}\right) d s\right), \\
& \operatorname{\varepsilon nergy}(\mathbf{P})=\frac{1}{2}\left(s+\int_{0}^{s}\left(\kappa_{g}^{2}+\tau_{g}^{2}\right) d s\right) \text {. }
\end{aligned}
$$

Proof. If we follow the similar steps as in the Theorem 4.4, the proof is obvious.

Case 3. Let $\mathbf{S}$ be an oriented timelike surface and moving particle lying on $\mathbf{S}$ has a unit timelike tangent vector $\mathbf{e}_{(0)}$.

Theorem 4.6. Let $\Gamma$ be a moving particle on surface $S$ such that it corresponds to a curve $\zeta$. Then, energy on the particle in tangent vector field by using Sasaki metric is stated by

$$
\text { Energye }_{(0)}=\frac{1}{2}\left(-s+\int_{0}^{s}\left(\kappa_{g}^{2}+\kappa_{n}^{2}\right) d s\right) .
$$

Proof. From Equation 3.1 and 3.2 we know

$$
\operatorname{\varepsilon nergye}_{(0)}=\frac{1}{2} \int_{0}^{s} \rho_{S}\left(d \mathbf{e}_{(0)}\left(\mathbf{e}_{(0)}\right), d \mathbf{e}_{(0)}\left(\mathbf{e}_{(0)}\right)\right) d s .
$$

By using the Equation 3.3 we have

$$
\begin{gathered}
\rho_{S}\left(d \mathbf{e}_{(0)}\left(\mathbf{e}_{(0)}\right), d \mathbf{e}_{(0)}\left(\mathbf{e}_{(0)}\right)\right)=\rho\left(d \omega\left(\mathbf{e}_{(0)}\left(\mathbf{e}_{(0)}\right)\right), d \omega\left(\mathbf{e}_{(0)}\left(\mathbf{e}_{(0)}\right)\right)\right) \\
+\rho\left(Q\left(\mathbf{e}_{(0)}\left(\mathbf{e}_{(0)}\right)\right), Q\left(\mathbf{e}_{(0)}\left(\mathbf{e}_{(0)}\right)\right)\right) .
\end{gathered}
$$

Since $\mathbf{e}_{(0)}$ is a section, we also get

$$
d(\omega) \circ d\left(\mathbf{e}_{(0)}\right)=d\left(\omega \circ \mathbf{e}_{(0)}\right)=d\left(i d_{C}\right)=i d_{T C} .
$$

Moreover, it is clear that

$$
Q\left(\mathbf{e}_{(0)}\left(\mathbf{e}_{(0)}\right)\right)=D_{\mathbf{e}_{(0)}} \mathbf{e}_{(0)}=\kappa_{g} \mathbf{P}+\kappa_{n} \mathbf{n} .
$$

Thus, we find from Equation 2.3

$$
\begin{aligned}
\rho_{S}\left(d \mathbf{e}_{(0)}\left(\mathbf{e}_{(0)}\right), d \mathbf{e}_{(0)}\left(\mathbf{e}_{(0)}\right)\right) & =\rho\left(\mathbf{e}_{(0)}, \mathbf{e}_{(0)}\right)+\rho\left(D_{\mathbf{e}_{(0)}} \mathbf{e}_{(0)}, D_{\mathbf{e}_{(0)}} \mathbf{e}_{(0)}\right) \\
& =-1+\kappa_{g}^{2}+\kappa_{n}^{2}
\end{aligned}
$$


and finally

$$
\text { Energye }_{(0)}=\frac{1}{2}\left(-s+\int_{0}^{s}\left(\kappa_{g}^{2}+\kappa_{n}^{2}\right) d s\right)
$$

Conclusion 4.4. Let $\zeta$ be a timelike curve lying on timelike surface $\mathbf{S}$. Then we have $\left\|D_{\mathbf{e}_{(0)}} \mathbf{e}_{(0)}\right\|^{2}=\kappa_{g}^{2}+\kappa_{n}^{2}$. Thus, we obtain following relation for the bending energy of elastica:

$$
H_{B}=\text { energye }_{(0)}+\frac{1}{2} s
$$

Proof. It is obvious from the Equation 4.1 and Theorem 4.6.

Theorem 4.7. Energy on the moving particle in surface's normal and tangent's normal vector field by using Sasaki metric is stated by

$$
\begin{aligned}
& \operatorname{\varepsilon nergy}(\mathbf{n})=\frac{1}{2}\left(-s+\int_{0}^{s}\left(-\kappa_{n}^{2}+\tau_{g}^{2}\right) d s\right) \text {, } \\
& \operatorname{\varepsilon nergy}(\mathbf{P})=\frac{1}{2}\left(-s+\int_{0}^{s}\left(-\kappa_{g}^{2}+\tau_{g}^{2}\right) d s\right) \text {. }
\end{aligned}
$$

Proof. If we follow the similar steps as in the Theorem 4.6, the proof is obvious.

\section{Conclusion}

In this study, we studied energy on the particle in the Darboux vector fields in Minkowski spacetime considering kinematics of the particle. Furthermore, we set a connection between energy on the particle in these vector fields and elastica of bending functional. This is important for our future work since a simple characterization on the energy of a vector field can be described as it is up to constants, in other words, it is square $L_{2}$ norm of the vector field's covariant derivative. Thanks to this definition, not only we will correlate the concept of the energy with a volume for the moving particle in these vector fields in space.

As is known, elastic energy may occur by applying different forces besides bending such as twisting and stretching. In our next studies, we also determine the correlation between energy on the particle in each Darboux vector field and stretching and twisting energy functional.

Computing the energy on the moving particle has a wide range of application in the theoretical and applied physics. Therefore, it will also be investigated the energy on the moving particle in different force fields thanks to classical mechanics by obtaining dynamics of the particle in space including work done and force acting on the particle besides the energy. We believe that this study also will lead up to further research on the relativistic dynamics of the particle in different spacetimes in terms of computing the energy on a particle in different force fields. 


\section{REFERENCES}

[1] C.M. Wood, On the Energy of a Unit Vector Field, Geom. Dedicata. 64 (1997), 319-330.

[2] O. Gil Medrano, Relationship between volume and energy of vector fields, Differ. Geom. Appl. 15 (2001) 137-152.

[3] P.M. Chacon, A.M. Naveira and J.M. Weston, On the Energy of Distributions, with Application to the Quaternionic Hopf Fibrations, Monatsh. Math. 133 (2001) 281-294.

[4] P.M. Chacon and A.M. Naveira, Corrected Energy of Distribution on Riemannian Manifolds, Osaka J. Math. 41 (2004) 97-105.

[5] A. Altin, On the energy and Pseduoangle of Frenet Vector Fields in $R_{v}$ ?, Ukr. Math J. 63 (2011) 969-975.

[6] G. Kirchhoff, ber Das Gleichgewicht und die Bewegung einer elastichen Scheibe, Crelles J. 40 (1850) 51-88.

[7] E. Catmull and J. Clark, Recursively generated b-spline surfaces on arbitrary topological surfaces, Comput.-Aided Des. 10 (1978), 350-355.

[8] T. Lopez-Leon, V. Koning, K.B.S. Devaiah, V. Vitelli and A.A. Fernandez-Nieves, Frustrated nematic order in spherical geometries, Nature Phys. 7 (2011) 391-394.

[9] T. Lopez-Leon, A.A. Fernandez-Nieves, M. Nobili and C. Blanc, Nematic-Smectic Transition in Spherical Shells, Phys. Rev. Lett. 106 (2011) 247802.

[10] J. Guven J, D.M. Valencia and J. Vazquez-Montejo, Environmental bias and elastic curves on surfaces, Phys. A: Math. Theory. 47 (2014) Article ID 355201.

[11] L. Euler, Additamentum 'de curvis elasticis', in Methodus Inveniendi Lineas Curvas Maximi Minimive Probprietate Gaudentes, Lausanne, 1744.

[12] C.H. Sequin, CAD Tools for Aesthetic Engineering, Comput.-Aided Des. Appl. 1 (2004) 301-309.

[13] D. Zorin, Curvature-based energy for simulation and variational modelling, Proceedings of the International Conference on Shape Modelling and Applications. SMI'05 (2005) 196-204.

[14] P. Joshi and C. Sequin, Energy Minimizer for Curvature-Based Surface Functional, CAD Conference, Waikiki, Hawaii. (2007) 607-617.

[15] A. Einstein, Zur Elektrodynamik bewegter K?rper, Annalen der Physik. 17 (1905), 891-921.

[16] A. Einstein, Relativity:The Special and General Theory, Henry Holt, New York, 1920.

[17] T. Roberts, S. Schleif and J.M. Dlugosz, What is the experimental basis of Special Relativity? Usenet Physics FAQ, 2007.

[18] A. Einstein, Does the inertia of a body depend on its energy content?, Annalen der Physik, 18 (1905) 639-641.

[19] M.K. Saad, H.S. Abdel-Aziz, G. Weiss and M.A. Soliman, Relation among Darboux frames of null Bertrand curves in Pseudo-Euclidean space, 1st Int. WLGK11, 2011.

[20] R. Capovilla, C. Chryssomalakos and J. Guven, Hamiltonians for curves, J. Phys. A. 35 (2002) 6571-6587.

[21] M. Carmeli, Motion of a charge in a gravitational field, Phys. Rev. B. 138 (1965) 1003-1007.

[22] J. Weber, Relativity and Gravitation, Interscience, New York, 1961.

[23] G. Napoli, L. Vergori, Extrinsic Curvature Effects on Nematic Shells, Phys. Rev. Lett. 108 (2012), Article ID 207803. 